\title{
Corrigendum: Identification of a role for TRIM29 in the control of innate immunity in the respiratory tract
}

Junji Xing, Leiyun Weng, Bin Yuan, Zhuo Wang, Li Jia, Rui Jin, Hongbo Lu, Xian Chang Li, Yong-Jun Liu \& Zhiqiang Zhang Nat. Immunol.; doi:10.1038/ni.3580; corrected online 10 October 2016

In the version of this article initially published online, the immunoblots in Figures $1 \mathrm{a}, 5 \mathrm{a}, 5 \mathrm{~b}, 5 \mathrm{c}, 5 \mathrm{~d}, 5 \mathrm{f}, 6 \mathrm{a}, 6 \mathrm{~b}, 7 \mathrm{a}, 7 \mathrm{~b}, 8 \mathrm{a}$ and $8 \mathrm{~b}$ were in color. These have been replaced with black and white immunoblots. The error has been corrected for the print, PDF and HTML versions of this article.

\section{Corrigendum: Evidence of innate lymphoid cell redundancy in humans}

Frédéric Vély, Vincent Barlogis, Blandine Vallentin, Bénédicte Neven, Christelle Piperoglou, Thibaut Perchet, Maxime Petit, Nadia Yessaad, Fabien Touzot, Julie Bruneau, Nizar Mahlaoui, Nicolas Zucchini, Catherine Farnarier, Gérard Michel, Despina Moshous, Stéphane Blanche, Arnaud Dujardin, Hergen Spits, Jörg H W Distler, Andreas Ramming, Capucine Picard, Rachel Golub, Alain Fischer \& Eric Vivier

Nat. Immunol. 17, 1291-1299 (2016); published online 12 September 2016; corrected after print 19 October 2016

In the version of this article initially published, author Mikael Ebbo was missing from the author list. The correct list is as follows: Frédéric Vély ${ }^{1,2,20}$, Vincent Barlogis ${ }^{3,20}$, Blandine Vallentin ${ }^{3,20}$, Bénédicte Neven ${ }^{4-7,20}$, Christelle Piperoglou ${ }^{1,2}$, Mikael Ebbo ${ }^{1,8}$, Thibaut Perchet ${ }^{9,10}$, Maxime Petit ${ }^{9,10}$, Nadia Yessaad ${ }^{11}$, Fabien Touzot 5 ,12, Julie Bruneau ${ }^{5,13}$, Nizar Mahlaoui ${ }^{4-7}$, Nicolas Zucchini ${ }^{14}$, Catherine Farnarier ${ }^{2}$, Gérard Michel ${ }^{3}$, Despina Moshous $^{4-7}$, Stéphane Blanche ${ }^{4-7}$, Arnaud Dujardin ${ }^{15}$, Hergen Spits ${ }^{16}$, Jörg H W Distler ${ }^{17}$, Andreas Ramming ${ }^{17}$, Capucine Picard ${ }^{4-7,18}$, Rachel Golub ${ }^{9,10}$, Alain Fischer ${ }^{4-7,19,21}$ \& Eric Vivier ${ }^{1,2,21}$. The correct affiliation list ends as follows: ${ }^{8}$ APHM, Hôpital de la Timone, Service de Médecine Interne, Marseille, France. ${ }^{9}$ Institut Pasteur, Unité de Lymphopoièse, INSERM, Paris, France. ${ }^{10}$ Université Paris Diderot, Sorbonne Paris Cité, Cellule Pasteur, Paris, France. ${ }^{11}$ MI-mAbs consortium, Aix-Marseille University, Marseille, France. ${ }^{12}$ APHP, Hôpital Necker-Enfants Malades, Biotherapy Unit, Paris, France. ${ }^{13}$ APHP, Hôpital Necker-Enfants Malades, Service d'anatomopathologie, Paris, France. ${ }^{14}$ BD Biosciences, Le Pont-de-Claix, France. ${ }^{15}$ Innate-Pharma, Marseille, France. ${ }^{16}$ Academic Medical Center at the University of Amsterdam, Arizona Amsterdam, the Netherlands. ${ }^{17}$ Department of Internal Medicine, Rheumatology \& Immunology, University of Erlangen-Nuremberg, Erlangen, Germany. ${ }^{18}$ APHP, Hôpital Necker-Enfants Malades, Study Center of Immunodeficiencies, Paris, France. ${ }^{19}$ College de France, Paris, France. ${ }^{20}$ These authors contributed equally to this work. ${ }^{21}$ These authors jointly directed this work. The correct Author Contributions section ends as follows: “...and M.E., N.M., N.Z., C.F., G. M., D.M., S.B., A.D., H.S. and C. Pic. provided key expertise, reagents or samples."

In addition, the description of patient C9 was incorrect in the text and legend for Figure 4b,d. The correct text (in the final paragraph of the fourth subsection of Results) is as follows: "In addition, NKp $46^{+}$ILCs and ILC2s were readily observed in tissues from patients treated with myeloablative HSCT, as illustrated by the analysis of skin biopsies from a patient with SCID who had mutation of RAG2 (C10) and was treated with myeloablative HSCT (Fig. 4b,d).... As a control, tissue-resident gut ILCs were observed in a patient with SCID who had mutation of RAG1 (C9) but was treated with 'pheno-related' HSCT (related donor with more than one compatible HLA haplotype but not genetically identical) under non-myeloablative conditions (Fig. 4b,d). The correct Figure 4b,d legend is as follows: “(b) Microscopy of tissue sections from patients who were treated with HSCT (stained as in a): duodenum (left) or colon (right) from C9 (RAG1 mutation) and skin from C10 (RAG2 mutation) (middle), at 15 months (C9) or 4 months (C10) after HSCT....(d) Microscopy of tissue sections from patients with SCID (colon from P5 and skin from P11 (as in a); colon from C9 and skin from C10 (as in b))..."

Also, a label was missing under Figure 1d, far left; that should be labelled as ' $\mathrm{Lin}^{-} \mathrm{CD} 127^{+}$.

Finally, the description of lung NK cells in the final paragraph of Results was incorrect; that text should read as follows: "Similarly, lung NK cells $\left(\mathrm{Lin}^{-} \mathrm{NKp} 46^{+} \mathrm{NK} 1.1^{+} \mathrm{CD} 127^{-}\right)$underwent reconstitution..." These errors have been corrected in the PDF and HTML versions of this article.

\section{Erratum: USP15 regulates type I interferon response and is required for pathogenesis of neuroinflammation}

Sabrina Torre, Maria J Polyak, David Langlais, Nassima Fodil, James M Kennedy, Irena Radovanovic, Joanne Berghout, Gabriel A Leiva-Torres, Connie M Krawczyk, Subburaj Ilangumaran, Karen Mossman, Chen Liang, Klaus-Peter Knobeloch, Luke M Healy, Jack Antel, Nathalie Arbour, Alexandre Prat, Jacek Majewski, Mark Lathrop, Silvia M Vidal \& Philippe Gros Nat. Immunol.; doi:10.1038/ni.3581; corrected online 24 October 2016

In the version of this article initially published online, the symbol for the gene encoding granzyme B was incorrect (Gmzb) in the text in the third paragraph of the fourth subsection of Results and Figure $5 \mathrm{~d}$, and the symbol for the gene encoding granzyme A was incorrect (Gmza) in Figure 6h. These should be Gzmb and Gzma, respectively. The errors have been corrected for the print, PDF and HTML versions of this article. 